\title{
Increased methylation of glucocorticoid receptor gene (NR3C1) in adults with a history of childhood maltreatment: a link with the severity and type of trauma
}

\author{
N Perroud ${ }^{1}$, A Paoloni-Giacobino ${ }^{2}$, P Prada ${ }^{3}$, E Olié4,5,6, A Salzmann ${ }^{1}$, R Nicastro ${ }^{3}$, S Guillaume ${ }^{4,5,6}$, D Mouthon ${ }^{2}$, \\ C Stouder ${ }^{2}, K_{\text {Dieben }}^{3}$, P Huguelet ${ }^{3}, P$ Courtet $^{4,5,6}$ and A Malafosse ${ }^{1,2}$
}

Childhood maltreatment, through epigenetic modification of the glucocorticoid receptor gene (NR3C1), influences the hypothalamic-pituitary-adrenal axis (HPA axis). We investigated whether childhood maltreatment and its severity were associated with increased methylation of the exon $1_{\mathrm{F}}$ NR3C1 promoter, in 101 borderline personality disorder (BPD) and 99 major depressive disorder (MDD) subjects with, respectively, a high and low rate of childhood maltreatment, and 15 MDD subjects with comorbid post-traumatic stress disorder (PTSD). Childhood sexual abuse, its severity and the number of type of maltreatments positively correlated with $N R 3 C 1$ methylation $\left(P=6.16 \times 10^{-8}, 5.18 \times 10^{-7}\right.$ and $1.25 \times 10^{-9}$, respectively). In BPD, repetition of abuses and sexual abuse with penetration correlated with a higher methylation percentage. Peripheral blood might therefore serve as a proxy for environmental effects on epigenetic processes. These findings suggest that early life events may permanently impact on the HPA axis though epigenetic modifications of the NR3C1. This is a mechanism by which childhood maltreatment may lead to adulthood psychopathology.

Translational Psychiatry (2011) 1, e59; doi:10.1038/tp.2011.60; published online 13 December 2011

\section{Introduction}

Exposure to early life adverse events, including abuses and neglect, significantly increases the risk of psychopathology in adulthood. .,2 $^{1,2}$

In humans as well as in animals, response to stress, and particularly to childhood trauma, is mediated by the hypothalamic-pituitary-adrenal (HPA) axis, which includes release of corticotrophin-releasing factor and secondarily results in secretion of glucocorticoids. ${ }^{3}$ Early life stress has been shown to induce persistent changes in corticotrophin-releasing factor neurotransmission and sustains alteration of the HPA axis. ${ }^{4,5}$ Hyperactivity as well as decreased activity of the HPA axis in response to stress has been demonstrated in humans with a history of childhood maltreatment. ${ }^{4-6}$ It has been speculated that the direction of such HPA alterations may depend on the characteristics of the stressor such as the type, duration, intensity or timing. ${ }^{7,8}$ Nevertheless, although the main role of this HPA axis is to prevent over-response to stress, ${ }^{9}$ prolonged exposure to elevated levels of glucocorticoids has damaging effects on the developing brain, which may secondarily lead to behavioral problems later in life. ${ }^{10-13}$

Recent studies point to the epigenetic mechanisms as a process by which environmental factors may alter the gene expression involved in control of the HPA axis. In rodents, epigenetic modification of specific exon $1_{7}$ in the promoter region of the glucocorticoid receptor (GR) gene (NR3C1) has been linked to early maternal care and secondarily to effects on hippocampal GR expression. ${ }^{14,15}$ In rats, Weaver et al. ${ }^{14}$ showed that this promoter region of $N R 3 C 1$ is regulated through early experience including the quality of maternal care. This epigenetic programming of GR expression inhibits the activation of HPA activity, ${ }^{16}$ which may in part explain how early maternal care influences HPA axis stress response in the offspring. ${ }^{14,17}$

To our knowledge, only three studies performed in humans to date-and only one of them in adults-have investigated the relationship between methylation status of the exon $1_{F}$ $N R 3 C 1$ promoter, the human homolog of the rat exon $1_{7}$ $N R 3 C 1$ promoter and early life adverse events. All three reached the same conclusion: higher methylation status was found in subjects who experienced early life adverse events. ${ }^{18-20}$ McGowan et al. ${ }^{19}$ showed increased methylation of the exon $1_{F} N R 3 C 1$ promoter in the hippocampus of suicide victims with a history of childhood abuse compared with control samples, which was associated with decreased levels of GR mRNA, as well as mRNA transcripts bearing the GR $1_{F}$ splice variant. Neither the hippocampal GR expression nor the methylation status of the exon $1_{F} N R 3 C 1$ promoter was altered in suicide victims with no history of abuse. This result suggests a specific effect of child abuse independent of

\footnotetext{
${ }^{1}$ Department of Psychiatry, University of Geneva, Chêne-Bourg, Switzerland; ${ }^{2}$ Department of Medical Genetic and Laboratories, University Hospitals of Geneva, Geneva, Switzerland; ${ }^{3}$ Department of Mental Health and Psychiatry, University Hospitals of Geneva, Geneva, Switzerland; ${ }^{4}$ INSERM U1061, Montpellier, France; ${ }^{5}$ University of Montpellier 1, Montpellier, France and ${ }^{6}$ Department of Emergency Psychiatry, University Hospital of Montpellier, Montpellier, France Correspondence: Dr N Perroud, Department of Psychiatry, University of Geneva, Hôpital de Belle-Idée, 2 ch. du Petit-Bel-Air, 1225 Chêne-Bourg, Switzerland. E-mail: nader.perroud@hcuge.ch

Keywords: borderline personality disorder; childhood maltreatment; epigenetic; methylation; NR3C1

Received 28 October 2011; accepted 5 November 2011
} 
suicide on methylation status and GR mRNA expression. Adding to the hypothesis of early environmental effect on NR3C1 epigenetic status in humans, Oberlander et al. ${ }^{18}$ found that prenatal exposure to increased third trimester maternal depressed/anxious mood was associated with increased methylation of NR3C1 in newborns (in genomic DNA from cord blood mononuclear cells) and increased salivary cortisol stress responses at 3 months. Finally, Radtke et al. ${ }^{20}$ showed an increased methylation of NR3C1 promoter in children exposed years earlier as a fetus to stress during their mother's pregnancy.

We undertook this study to examine whether childhood maltreatment, with a primary focus on childhood sexual abuse, was associated with increased NR3C1 promoter methylation in a sample of borderline personality disorder (BPD) subjects expected to have a high rate of childhood traumas (sexual, physical as well as emotional) and a sample of mildly abused and neglected subjects suffering from major depressive disorder (MDD) and a sample of subjects suffering from MDD with comorbid past/current PTSD. As some data moreover suggest that higher severity and type of abuse are risk factors for worse mental health outcome in adulthood ${ }^{21}$ (see the Supplementary Information for additional references), we also expected the severity of the maltreatment (intensity of the trauma, age at onset, number of type of abuses/neglects and their repetition) to be positively correlated with the percentage of methylation.

\section{Materials and methods}

Samples. A total of 101 subjects suffering from BPD were recruited in a specialized center using intensive dialectical behavior therapy as the core treatment for this disorder. ${ }^{22}$ Briefly, patients were referred by their physician or other medical services for suicidal or deliberate self-harm behaviors. All participants received psychopharmacological treatment, which was refined by a psychiatrist if necessary before and during follow-up. Blood monitoring to ascertain compliance was systematically done at entry in the program. All patients were assessed for Axis I psychiatric diagnoses using the French version of the Diagnostic Interview for Genetic Studies ${ }^{23}$ and for BPD by the Screening Interview for Axis II Disorders BPD part. ${ }^{24}$ Only subjects fulfilling DSM-IV criteria for BPD were accepted in the program. Each participant was interviewed by either a trained psychiatrist or a psychologist before acceptance into treatment. Before 1 week the commencement of intensive dialectical behavior therapy each subject completed the Beck Depression Inventory $\mathrm{II}^{25}$ to assess the current severity of depression symptoms and the Childhood Trauma Questionnaire (CTQ), which examines five types of trauma in a self-report assessment: sexual abuse, physical abuse, physical neglect, emotional abuse and emotional neglect. Scores range from 5 to 25 for each type of trauma. According to Bernstein and Fink, ${ }^{26}$ thresholds or cutoff scores have been set for each type of trauma at four levels of maltreatment: none, low, moderate and severe.

In addition to the CTQ, each participant was clinically questioned about the existence or not of sexual or physical abuses during childhood and adulthood. If abuses were reported, the following items were then systematically explored: age of the abuse, duration of the abuse, frequency of the abuse, perpetrator of the abuse, nature of the abuse and the number of abuses throughout life. Each case was then discussed with the team program, ${ }^{22}$ and a final report on abuse was then made. According to the literature, we classified the abuses as follows: ${ }^{21}$ frequency of abuses (day, month, week or year), number of consecutive abuses (repetition of abuses: one, two or more), relationship to the perpetrator (family member or not), child vs adolescent/ adulthood abuses, sexual abuse with or without penetration.

Demographic and additional clinical data were obtained from a standard questionnaire given to all participants before entering the program. These data were checked against notes from psychiatric, medical and surgical units.

As BPD subjects were expected to have a high rate of abuses and neglects (thus methylation in subjects with no maltreatment would be difficult to ascertain due to the ceiling effect) and to suffer from MDD and PTSD comorbidities, we decided to contrast our BPD sample with two other samples. First, 99 subjects with a low rate of abuses and neglect were selected from a cohort of $>1500$ subjects suffering from various psychiatric disorders and for which CTQ was available. These subjects were recruited from consecutive admissions to the psychiatric university hospitals of Montpellier (France) and Geneva (Switzerland) between 1994 and 2010. Subjects were assessed for psychiatric diagnoses using either the Diagnostic Interview for Genetic Studies or the Mini International Neuropsychiatric Interview. ${ }^{27}$ All of them fulfilled the CTQ and a part the Beck Depression Inventory short form (Beck Depression Inventory-Short Form; 13 items). All subjects with a diagnosis of MDD without past/current PTSD who reported no sexual abuse, no physical abuse and neglect and no emotional abuse were chosen. 'No emotional neglect' was not considered as an inclusion criterion, as only 49 subjects among the 1500 patients failed to report neglect of this kind in addition to failing to report any of the other abuses or neglect (see Table 1). Second, 15 MDD subjects with past/ current PTSD were chosen from the above sample of 1500 . The sole selection criteria were suffering from MDD and having a past/current diagnosis of PTSD based on the Diagnostic Interview for Genetic Studies.

The study protocol was approved by the Research Ethics Committees in each center, and written informed consent was obtained for each subject.

NR3C1 methylation status. For the BPD subject, blood samples were systematically collected 1 week before the commencement of the program. For MDD subjects blood samples were collected just after the interviews. DNA was extracted from blood white cells (Nucleon extraction kit, Amersham Life Science, Dubendorf, Switzerland).

We analyzed a portion of the exon $1_{\mathrm{F}}$ NR3C1 promoter, which was equivalent to the one reported by Oberlander et al. ${ }^{18}$ to exhibit methylation modulation in response to antenatal maternal mood and reported by McGowan et al. ${ }^{19}$ to exhibit methylation differences in suicide victims with a history of sexual abuse. It should be noted that this region 


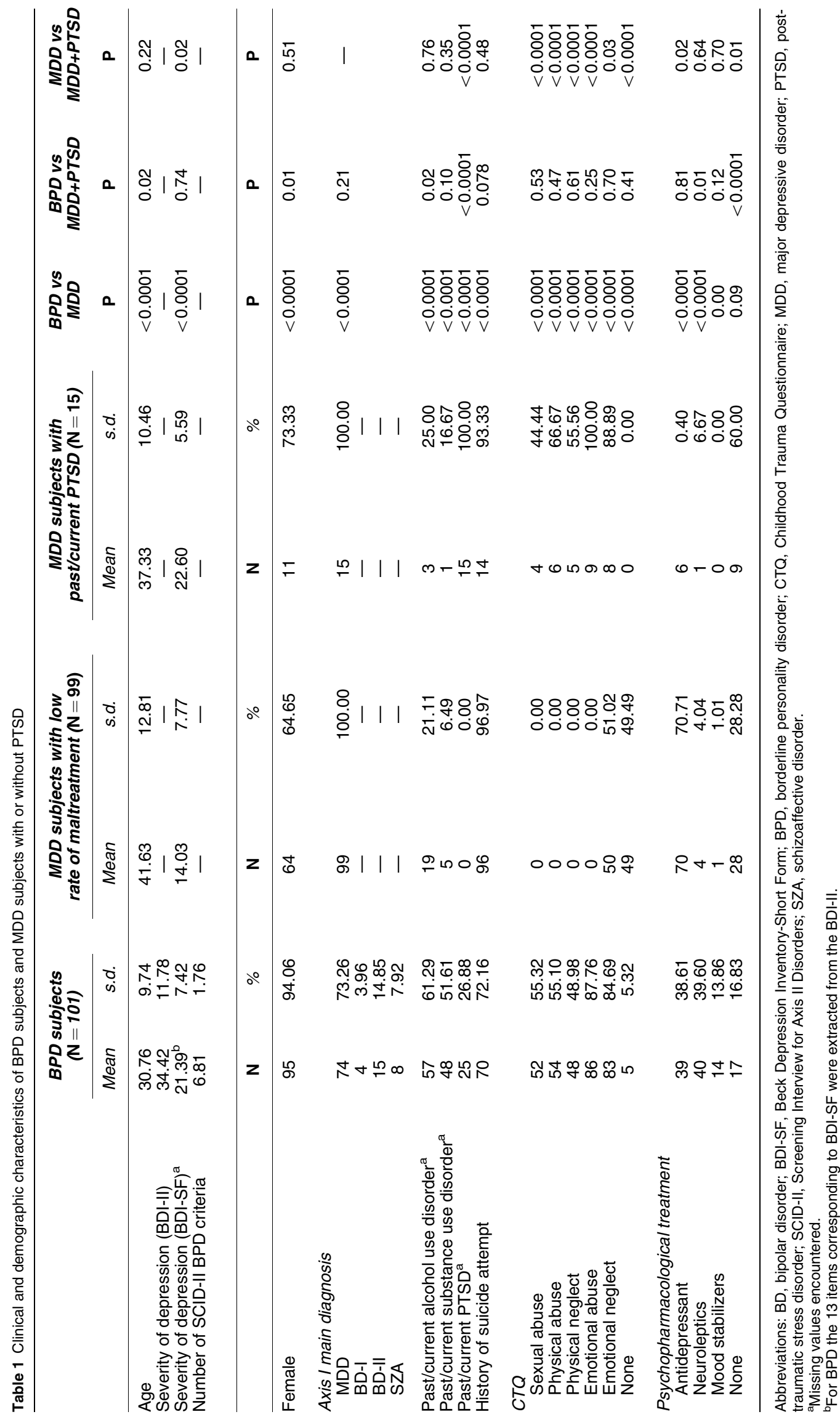






Figure 1 The region from nucleotide -3492 to -3082 (numbers relative to the translation start site considered as +1 ) of the $5^{\prime}$-end of the $N R 3 C 1$ gene. Underlined: exon 1F; square boxes: position of the oligonucleotides used for amplification of bisulfite-treated DNA; encircled: CpGs analyzed.

contains a nerve growth factor inducible protein A-binding site that regulates gene transcription.

The degree of methylation at each $\mathrm{CpG}$ site was determined using Pyro Q-CpG Software (Biotage AB, Uppsala, Sweden). Details of the bisulfite treatment and of the PCR amplification of bisulfite-treated DNA for subsequent pyrosequencing are available in the Supplementary Information.

Eight CpG sites were analyzed and the percentage of total methylation was calculated (Figure 1). These eight CpGs correspond to CpGs 6-13, described by Oberlander et al. ${ }^{18}$ and are located immediately downstream to those described by McGowan et al. ${ }^{19}$ CpG1 in our study is located 20 nucleotides downstream of what McGowan et al. described as CpG 39. All samples were analyzed in duplicate and the mean percentage was then calculated and used for the current analyses. All duplicates with $3 \%$ error or more between the two measures were either re-analyzed and used if $<3 \%$ error was then observed between the two new measures or were excluded from the analyses $(N=7)$ if the $3 \%$ error or more was still present. It should be noted that, in a previous study, the results obtained showing a small $(\leqslant 3 \%)$ difference in methylation in two difference conditions were double checked by comparing the technique used above, that is, DNA bisulfite treatment and PCR amplification followed directly by pyrosequencing, with PCR amplification followed by amplicon subcloning and sequencing. The results were found to be similar and confirmed that pyrosequencing is a technique permitting to detect small percentages of difference with sensitivity as good as the cloning sequencing technique. ${ }^{28}$ Samples were processed and analyzed blindly with respect to demographic variables, psychiatric diagnoses and childhood maltreatment history.

Statistics. The analyses were carried out in two sequential steps, starting with the better-characterized sample of BPD expected to be enriched for childhood trauma history and for which a large panel of potential confounding factors was available. Confounding factors (see below) were assessed for association with methylation status and with each of the childhood maltreatments using either the linear regression or the $\chi^{2}$ test, respectively. Any variable showing an association reaching a $P$-value of 0.05 or below was then used as a covariate in a linear regression model. Methylation status was used without any transformation as it was normally distributed, in the BPD and the whole sample, according to the one-sample Kolmogornov-Smirnov test.

As type of childhood trauma has previously been shown to be an important correlate of adult psychopathology, ${ }^{18}$ each type of maltreatment as measured by the CTQ (sexual abuse, emotional and physical abuses/neglects) was first analyzed separately as a binary outcome (yes or no). We started the analyses with childhood sexual abuse as this has been the most consistently and most widely investigated childhood maltreatment. ${ }^{18}$ If significantly associated with methylation status we then ascertained whether the severity of the individual trauma (using CTQ cutoff scores described above) as a continuous variable was associated with methylation status. Second, and based on evidences from the literature ${ }^{29,30}$ we investigated whether the number of types of childhood maltreatment (by summing the different maltreatments reported by the CTQ) as a continuous variable was associated with methylation status. Finally, and only for the BPD sample, association between clinically recorded abuses and methylation status was tested.

The same methodology was then applied to the whole sample (MDD + BPD samples). Results of regression models are presented as standardized regression coefficients (b), which can be interpreted as effect size; however, values in the text represent the mean percentage of methylation with the corresponding s.d. The threshold of significance was set at $P \leqslant 0.05$.

\section{Results}

BPD sample. Table 1 displays the clinical and demographic characteristics of the BPD subjects. Most of them were female $(94.06 \%)$, aged around 30 years old (30.76, s.d. $=9.76)$, suffering from comorbid MDD $(73.26 \%)$, and from alcohol $(61.3 \%)$ and substance use disorders $(51.6 \%)$. 
One fourth of the BPD patients presented a diagnosis of current or past PTSD. The mean number of BPD DSM-IV items was 6.81 (s.d. $=1.76$ ) as measured by the Screening Interview for Axis II Disorders. Only 5 out of the 101 BPD subjects reported no childhood maltreatment.

Childhood maltreatment, methylation status and potential confounding factors. There was no association between methylation status and any of the potential clinical and demographic confounding factors. Gender, primary Axis I diagnosis, past/current alcohol/substance use disorder, past/current PTSD, history of suicide attempt, severity of depression and of BPD and current pharmacological treatment were all associated with childhood maltreatment to a certain degree and with childhood sexual abuse in the majority of cases (see Supplementary Information). These variables were therefore used as covariates in the analyses.

Effect of childhood maltreatment on NR3C1 methylation. There was a significant association between childhood sexual abuse and methylation status, with sexually abused subjects being more highly methylated $(0.141$ $($ s.d. $=0.02)$ ) than non-sexually abused subjects $(0.128$ (s.d. $=0.02) ; b=0.41 ; P=0.011$ ). The association was still significant after adjusting for all the significant confounding factors $(b=0.45 ; \quad P=0.015)$. After adjustment for confounding factors, increased severity of childhood sexual abuse was significantly associated with increased $N R 3 C 1$ methylation (none $=0.128$ (s.d. $=0.02$ ); low $=0.138$ (s.d. =0.03); moderate $=0.148$ (s.d. $=0.02$ ); severe $=0.137$ (s.d. $=0.02) ; b=0.13 ; P=0.039$; Figure 2a).

There was no association between childhood physical abuse and methylation status.

There was a significant association between childhood physical neglect and methylation status, with physically neglected subjects being more highly methylated $(0.141$ (s.d. =0.02)) than non-physically neglected subjects $(0.129$ (s.d. $=0.02) ; b=0.38 ; P=0.017$ ). The association was still significant after adjusting for all the significant confounding factors ( $b=0.41 ; P=0.015$; Supplementary Figure $\mathrm{S} 1$ ).

There was no association between childhood emotional abuse or neglect and methylation status.

After adjustment for confounding factors, there was a significant association between the number of types of childhood abuse and neglect and methylation status ( $b=0.12 ; P=0.034$; Figure $3 a$ ).

After adjustment for confounding variables, there was a significant trend in percentage of methylation from no clinically recorded childhood sexual abuse $(0.131$ (s.d. $=0.02)$ ) to sexual abuse without penetration $(0.137$ (s.d. $=0.02))$ and to sexual abuse with penetration (0.141 (s.d. $=0.01$ ); $b=0.22$; $P=0.02)$. The number of abuses over a subject's life span (repetition of abuse) was also significantly associated with methylation status after adjustment for confounding factors. Those with one reported abuse had less methylation $(0.131$ (s.d. $=0.02)$ ) than those reporting two or more abuses $(0.141$ (s.d. $=0.02)(b=0.28 ; P=0.043)$. (See Table 2 for details on the clinical evaluation of physical/sexual abuse).

Whole sample. Almost all the clinical and demographic variables distinguishing BPD subjects from MDD subjects (see Table 1) were significantly associated with methylation status and were therefore used as covariates in the following analyses (see the Supplementary Information).

Effect of childhood maltreatment on NR3C1 methylation. There was a significant association between sexual abuse and NR3C1 methylation $(b=1.10$; $P=1.98 \times 10^{-12}$ ) showing that sexually abused subjects had higher methylation status $(0.138$ (s.d. $=0.02))$ than
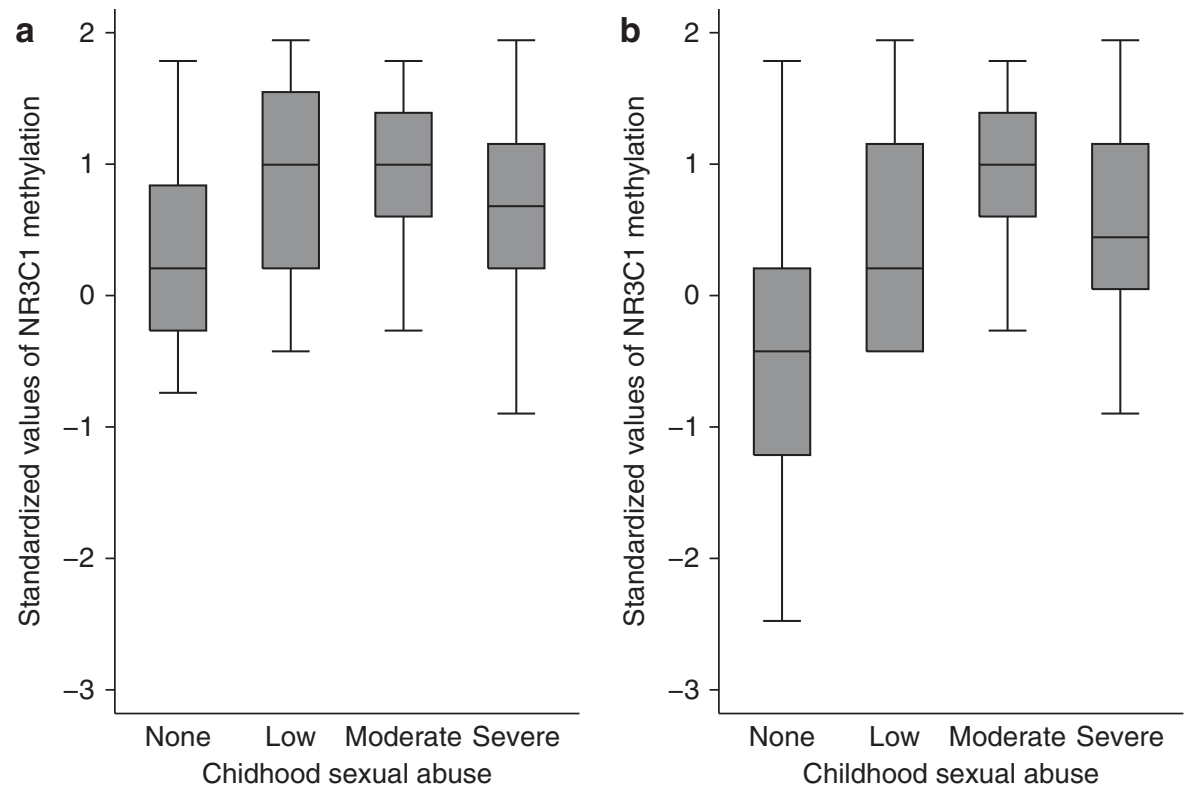

Figure 2 Box-plot of standardized methylation rate according to severity of sexual abuse in borderline personality disorder (BPD) subjects (a) and in the whole sample (b). 

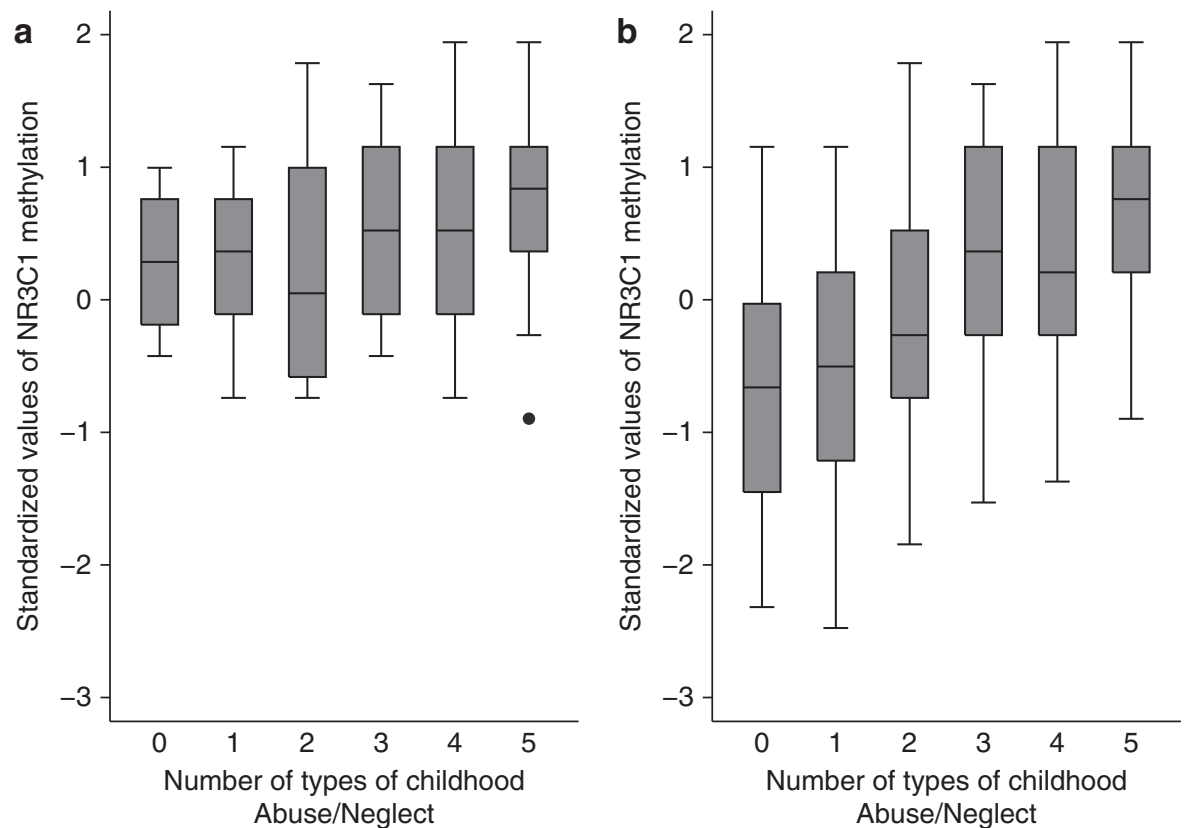

Figure 3 Box-plot of standardized methylation frequency according to the number of types of childhood abuse and neglect in BPD subjects (a) and in the whole sample (b).

Table 2 Evaluation of abuse in 75 physically and sexually abused borderline personality disorder (BPD) subjects

\begin{tabular}{|c|c|c|}
\hline & Mean & s.d. \\
\hline \multirow[t]{2}{*}{ Duration of abuses (years) } & $\begin{array}{l}6.93 \\
7.03\end{array}$ & $\begin{array}{l}5.31 \\
6.37\end{array}$ \\
\hline & $N$ & $\%$ \\
\hline \multicolumn{3}{|l|}{ Frequency of abuses } \\
\hline Day & 11 & 15.07 \\
\hline Month & 15 & 20.55 \\
\hline Week & 31 & 42.47 \\
\hline Year & 16 & 21.92 \\
\hline \multicolumn{3}{|l|}{ Number of consecutive abuses } \\
\hline 1 & 38 & 50.67 \\
\hline 2 & 30 & 40 \\
\hline 3 or more & 7 & 9.33 \\
\hline \multicolumn{3}{|l|}{ Perpetrator of the first abuse } \\
\hline First-degree relatives & 47 & 62.66 \\
\hline Second-degree relatives & 11 & 14.66 \\
\hline Close relatives (friends, neighbor) & 13 & 17.33 \\
\hline Others (priest, doctor, unknown) & 4 & 5.33 \\
\hline \multicolumn{3}{|l|}{ Perpetrator of the second abuse $(N=36)$} \\
\hline First-degree relatives (including husbands) & 10 & 27.78 \\
\hline Second-degree relatives & 3 & 8.33 \\
\hline Close relatives (friends, neighbor) & 16 & 44.44 \\
\hline Others (priest, doctor, unknown) & 7 & 19.44 \\
\hline \multicolumn{3}{|l|}{ Childhood sexual abuse ( $<14$ years old) } \\
\hline Without penetration & 19 & 25.33 \\
\hline With penetration (oral, vaginal or anal) & 17 & 22.66 \\
\hline \multicolumn{3}{|c|}{ Adolescence/Adulthood sexual abuse ( $\geqslant 14$ years old) } \\
\hline Without penetration & 5 & 6.66 \\
\hline With penetration (oral, vaginal or anal) & 24 & 32 \\
\hline Childhood physical abuse & 46 & 61.33 \\
\hline Adolescence/Adulthood physical abuse & 23 & 30.66 \\
\hline
\end{tabular}

non-sexually abused subjects $(0.103 \quad$ (s.d. $=0.03)$; Figure 2b). The association was still significant after adjustment for all the significant confounding factors $\left(b=1.01 ; \quad P=6.16 \times 10^{-8}\right)$. After adjustment for confounding factors, there was a significant trend from no childhood sexual abuse $(0.103$ (s.d. $=0.03))$, to low severity of sexual abuse $(0.132$ (s.d. $=0.03))$ to moderate severity $(0.147$ (s.d. $=0.02))$ and to severe sexual abuse $(0.134$ (s.d. =0.02); $b=0.36, P=5.18 \times 10^{-7}$ ).

Interestingly, when Figure $2 \mathrm{a}$ is compared with Figure $2 \mathrm{~b}$ it is obvious that, after adjustment for confounding factors, there is a significant difference in methylation status between BPD subjects and MDD subjects when those without sexual abuse are considered $\left(b=0.95 ; P=5.99 \times 10^{-7}\right)$.

There was a significant association between childhood physical abuse and methylation status $(b=0.79$; $\left.P=8.91 \times 10^{-7}\right)$. This association, which shows higher methylation in physically abused subjects $(0.131$ $($ s.d. $=0.02))$ than in non-physically abused subjects $(0.106$ (s.d. =0.03)), was still significant after adjusting for confounding factors $(b=0.61 ; P=0.003$; Supplementary Figure $S 1)$.

There was a significant association between physical neglect and NR3C1 methylation $\left(b=0.95 ; P=3.34 \times 10^{-9}\right)$ in the same direction as in the BPD sample $(0.135$ (s.d. $=0.03)$ vs 0.105 (s.d. =0.03); Supplementary Figure S1).

There was a highly significant association between childhood emotional abuse and NR3C1 methylation $(b=1.06$; $P=5.11 \times 10^{-15}$ ) showing that emotionally abused subjects had higher methylation status $(0.135($ s.d. $=0.03))$ than nonemotionally abused subjects $(0.098$ (s.d. $=0.03)$; Supplementary Figure S1). The association was still significant after adjusting for all the significant confounding factors $(b=1.18$; $P=7.24 \times 10^{-10}$ ). 


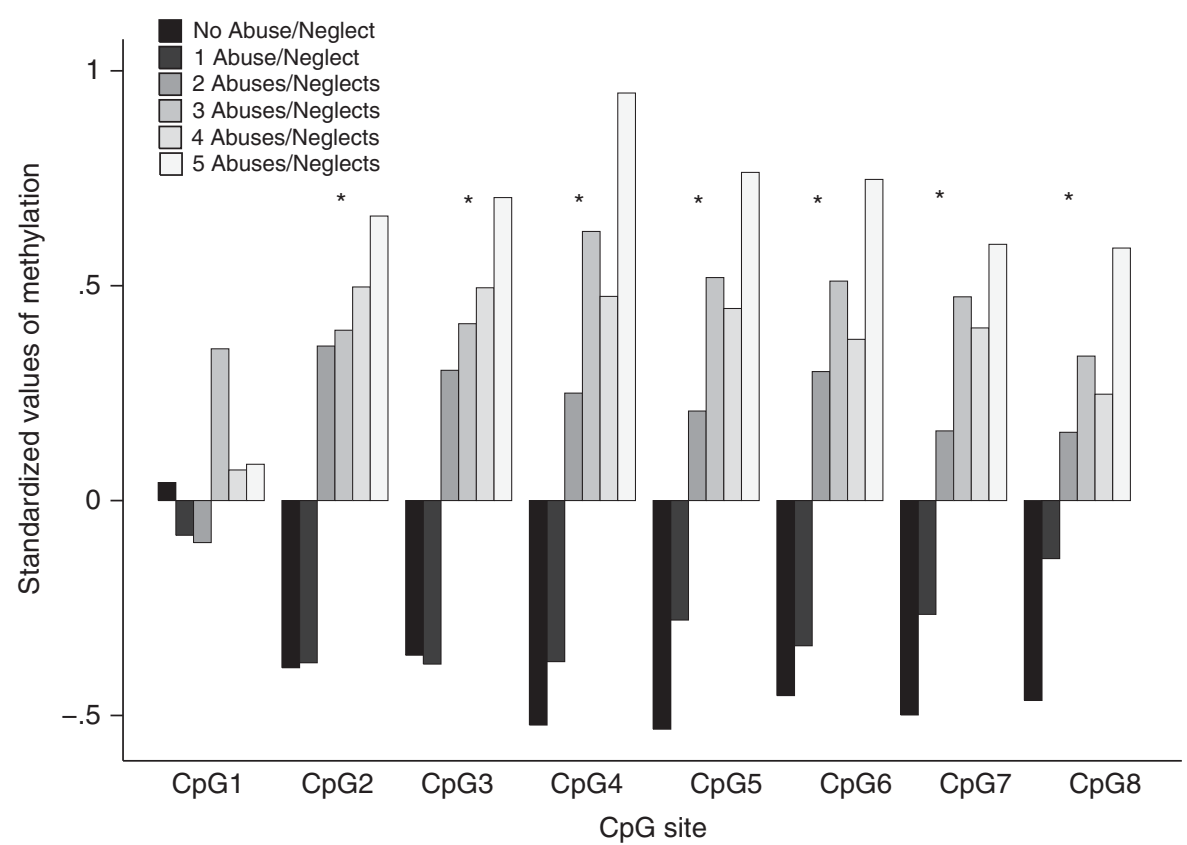

Figure 4 Methylation of the exon $1_{F} N R 3 C 1$ promoter region, showing the standardized value of methylation observed at each $\mathrm{CpG}$ site according to the number of types of childhood abuse and neglect in the whole sample. $\left({ }^{\star} P<1 \times 10^{-6}\right)$.

There was a significant association between childhood emotional neglect and methylation status $(b=0.56$; $P=0.0002)$ showing higher methylation in emotionally neglected subjects $(0.118$ (s.d. $=0.03))$ than in non-emotionally neglected subjects $(0.101(\mathrm{~s} . \mathrm{d} .=0.03))$ that was still significant after adjustment for confounding factors $(b=0.44$; $P=0.008)$. As is obvious from Supplementary Figure $\mathrm{S} 1$, emotional neglect positively associated with methylation status, high neglect predicting higher methylation status (from 0.101 (s.d. $=0.03$ ) to $0.130(0.03) P=2.71 \times 10^{-6}$ ).

There is an obvious association between the number of types of abuse and neglect and NR3C1 methylation status from 0.096 (s.d. $=0.03$ ) to 0.141 (s.d. $=0.02 ; \quad b=0.30$; $P=4.94 \times 10^{-15}$; cf. Figure $\left.3 b\right)$. This was still significant after adjustment for confounding factors $(b=0.31$; $P=1.25 \times 10^{-9}$ ).

With the exception of CpG1, all CpGs showed an association with the number of types of abuse and neglect in the same direction than the one observed for the whole methylation at a level of significance of $P<1 \times 10^{-6}$ (Figure 4).

\section{Discussion}

According to our primary hypothesis, we showed that childhood sexual abuse was associated with increased NR3C1 promoter methylation in the peripheral blood. Moreover, we also found that the severity of childhood abuses and neglect (repetition of abuses, number of types of abuse and neglect, types of abuses) positively correlated with NR3C1 promoter methylation.

In a recent paper, McGowan et al. ${ }^{19}$ showed that childhood sexual abuse was associated with increased methylation of the exon $1_{F} N R 3 C 1$ promoter in the brain of suicide victims. This paper was the first to highlight such an association opening the way for the next step: are we able to see a similar change in the peripheral blood of living patients in whom less recollection bias should come into play? Exploring the same region as McGowan et al., ${ }^{19}$ we suggest that what was primarily observed in the brain is true of the peripheral blood. We have added therefore to the growing body of evidence linking childhood maltreatment to the permanent alteration of the HPA axis and specifically here to epigenetic modifications of the NR3C1 promoter region. Glucocorticoids are the final effectors of the HPA axis and exert their activity in part via GR. The effect of negative feedback of glucocorticoids on adrenocorticotropic hormone secretion is to limit excessive glucocorticoid exposure. ${ }^{9}$ Interestingly, childhood abuse has been associated with an increased adrenocorticotropic hormone response to stress. ${ }^{31}$ It is hypothesized here that excessive methylation of the $N R 3 C 1$ promoter region as a consequence of childhood maltreatment permanently disturbs the expression and thus the availability of the GR in the brain and thus alters normal glucocorticoid negative feedback on adrenocorticotropic hormone. ${ }^{14,15,17}$ This long-lasting effect on the HPA axis is possibly one mechanism by which early adverse events may have an impact on adult psychopathology. ${ }^{3,5,31-34}$ Hypothetically, this is one mechanism linking childhood maltreatment to the development of adulthood BPD.

With the exception of CpG1, methylation at all the other CpGs showed an association with childhood maltreatment. The sites we explored were located a few nucleotides downstream of the specific ones reported by McGowan et al. $^{19}$ to have increased methylation levels in subjects with childhood sexual abuse. These results, as well as those obtained by Oberlander et al. ${ }^{18}$ could suggest that differences in methylation levels may occur only at specific sites in the exon $1_{F} N R 3 C 1$ promoter, sites located near the nerve growth 
factor inducible protein A recognition elements. This clearly requires investigation in further studies.

Our data showed that other forms of abuse and neglect, such as physical neglect, may have an effect on gene methylation and that the number of types of abuse/neglect strongly correlated with methylation percentage. Childhood maltreatment research has primarily focused on the effect of childhood sexual abuse and physical abuse whereas childhood emotional abuses and neglects as well as physical neglects have received little attention. Several studies are now suggesting that childhood emotional abuse may be as predictive of risk for adult psychopathology as sexual and physical abuse..$^{35}$ Interestingly, Teicher et al. ${ }^{29,30}$ have shown that exposure to multiple forms of maltreatment had a greater effect than additive deleterious effect.

The association between repetition of abuses and NR3C1 methylation status is of particular interest, as most of the data suggest that exposure to abuse and other early life events is associated with an increased risk for subsequent abuse. ${ }^{36}$ This raises the question as to whether NR3C1 epigenetic modifications are the cause or consequences of the abuses. Ideally, a sample consisting of subjects who were sexually abused or maltreated in their childhood but have no adult psychopathology may help to answer this question.

We showed that the type of sexual abuse (sexual abuse with or without penetration), and the severity of these abuses were associated with increased methylation status. It has been shown that the total brain volume positively correlated with age of onset of trauma-related PTSD and negatively correlated with the duration of abuse, ${ }^{37}$ suggesting that severity of abuse is a strong correlate of biological modification in humans. Some data have shown that sexual abuse with penetration, severity and certain type of abuses (the use of force or threats, longer duration and frequency of abuse, repetition of abuses) are linked to worse outcome in adulthood. ${ }^{21}$ Our data are therefore concordant with this literature and suggest that the degree of NR3C1 promoter methylation may be a marker of the severity of these abuses.

Surprisingly, BPD patients with no sexual abuse as well as those with no childhood maltreatment have higher NR3C1 methylation than MDD subjects with the same reported abuses and neglect in infancy. This could be related to the fact that BPD could be per se linked to higher methylation status than MDD alone, or that other environments were not carefully considered in this study, while explaining the higher percentage of methylation in this population. Maternal anxious/depressed mood during pregnancy has been shown to correlate with NR3C1 methylation in newborns. ${ }^{18}$ Moreover, maternal exposure to intimate partner violence during pregnancy predicted the methylation status of NR3C1 in adolescent children. ${ }^{20}$ The very early environment, which was not assessed in our study, may also explain higher methylation in BPD subjects. In the rodent model, variations in early maternal care are associated with differences in HPA stress response in the offspring and with epigenetic alterations in gene expression. ${ }^{14,17}$ Moreover, increased HPA stress reactivity in the offspring of mothers exhibiting decreased levels of maternal care appears to be mediated by decreased hippocampal NR3C1 expression. ${ }^{17,38}$ BPD subjects often have dysfunctional parents who sometimes suffer from BPD themselves. ${ }^{39}$ The hypothesis that BPD subjects have higher methylation status than MDD because of a very early adverse environment makes sense.

Although the severity of BPD correlated with sexual abuse (see Supplementary Information), it did not correlate with methylation percentage and thus we cannot conclude that methylation status is an indirect extrapolation of the severity of psychopathology in adulthood. The link may be more complex, as genes not explored in this study may be at play in the construction of this complex phenotype. ${ }^{40}$

Another limitation is that we used peripheral blood cells as study material. Although it has been shown that $N R 3 C 1$ exon $1_{F}$ is detected in peripheral blood (in CD19 $B$ lympocytes and plasmacytoid dendritic immune cells), ${ }^{41}$ this results and those from previous studies ${ }^{40}$ imply that childhood maltreatment modulates its methylation in the same way as in the brain. Study of methylations of the same CpGs in peripheral blood and brain tissues from the same subjects are required to confirm that peripheral blood may be used as a proxy for methylation processes occurring in the brain.

Finally, it remains to be demonstrated that $N R 3 C 1$ exon $1_{F}$ methylation is also associated with decreased transcription in peripheral blood, as previously shown by McGowan et al. ${ }^{40}$ in human brain.

Nevertheless, and as suggested by Philibert et al., ${ }^{42}$ major life stressors should impact both on peripheral and central tissues and result in correlated changes across multiple cell types. Despite these limitations, it is not unreasonable to hypothesize that our findings in peripheral blood might reflect changes in cell brains.

GR has an important role in multiple physiological and pathophysiological processes including response to glucocorticoids as treatment used in a number of inflammatory, autoimmune and neoplastic diseases. If and how the methylation processes observed in our study have an impact on peripheral GR functions, such as treatment response, requires further investigations.

This is the first human study to show an association between a wide range of childhood trauma and the methylation status of the promoter and exon $1_{\mathrm{F}}$ of the gene for GR (NR3C1) in peripheral blood in adults. Moreover, it shows for the first time the link between methylation status and the severity and type of childhood maltreatment. Although this result may be striking, it is in line with some data suggesting that the severity of childhood abuses is associated with poor mental health outcome in adulthood and indicating that the degree of NR3C1 promoter methylation may be a surrogate marker of maltreatment severity. The quality of parental care and of early life environment has developmental effects on brain development ${ }^{12}$ including disturbance of the HPA axis and exerts a broad impact on later mental health, including the risk of psychopathology. Our findings temptingly suggest that epigenetic processes might mediate the effects of these early environments on psychopathology into adulthood through effects on HPA axis activity.

\section{Conflict of interest}

The authors declare no conflict of interest. 
Acknowledgements. This study was supported by the SNF Grant \#320030-112084 and Grant UF 7653 CHU Montpellier \& ANR NEURO GENESIS. We thank Catherine Genty, Laury Anne Casse, Gérald Bouillault, Jean-Jaques Kunckler, Brigitte Blanchon, Venus Kaby, Christine Bourly and Catherine Angela Foglietta for the data collection.

1. Mullen PE, Martin JL, Anderson JC, Romans SE, Herbison GP. The long-term impact of the physical, emotional, and sexual abuse of children: a community study. Child Abuse Negl 1996; 20: 7-21.

2. McEwen BS. Early life influences on life-long patterns of behavior and health. Ment Retard Dev Disabil Res Rev 2003; 9: 149-154.

3. Meaney MJ. Maternal care, gene expression, and the transmission of individual differences in stress reactivity across generations. Annu Rev Neurosci 2001; 24: 1161-1192.

4. Heim C, Newport DJ, Heit S, Graham YP, Wilcox M, Bonsall R et al. Pituitary-adrenal and autonomic responses to stress in women after sexual and physical abuse in childhood. JAMA 2000; 284: 592-597.

5. De Bellis MD, Baum AS, Birmaher B, Keshavan MS, Eccard CH, Boring AM et al. A.E. Bennett Research Award. Developmental traumatology. Part I: Biological stress systems. Biol Psychiatry 1999; 45: 1259-1270.

6. Carpenter LL, Carvalho JP, Tyrka AR, Wier LM, Mello AF, Mello MF et al. Decreased adrenocorticotropic hormone and cortisol responses to stress in healthy adults reporting significant childhood maltreatment. Biol Psychiatry 2007; 62: 1080-1087.

7. Tyrka AR, Wier L, Price LH, Ross N, Anderson GM, Wilkinson CW et al. Childhood parental loss and adult hypothalamic-pituitary-adrenal function. Biol Psychiatry 2008; 63 . $1147-1154$

8. Mathew SJ, Coplan JD, Smith EL, Scharf BA, Owens MJ, Nemeroff CB et al Cerebrospinal fluid concentrations of biogenic amines and corticotropin-releasing factor in adolescent non-human primates as a function of the timing of adverse early rearing. Stress 2002; 5 185-193.

9. Carrasco GA, Van de Kar LD. Neuroendocrine pharmacology of stress. Eur J Pharmacol 2003; 463: 235-272.

10. Sapolsky RM, Uno H, Rebert CS, Finch CE. Hippocampal damage associated with prolonged glucocorticoid exposure in primates. J Neurosci 1990; 10: 2897-2902.

11. Teicher MH, Ito Y, Glod CA, Andersen SL, Dumont N, Ackerman E. Preliminary evidence for abnormal cortical development in physically and sexually abused children using EEG coherence and MRI. Ann NY Acad Sci 1997; 821: 160-175.

12. De Bellis MD, Keshavan MS, Clark DB, Casey BJ, Giedd JN, Boring AM et al. A.E. Bennett Research Award. Developmental traumatology. Part II: Brain development. Biol Psychiatry 1999; 45: 1271-1284.

13. Champagne DL, Bagot RC, van Hasselt F, Ramakers G, Meaney MJ, de Kloet ER et al. Maternal care and hippocampal plasticity: evidence for experience-dependent structura plasticity, altered synaptic functioning, and differential responsiveness to glucocorticoids and stress. J Neurosci 2008; 28: 6037-6045.

14. Weaver IC, Cervoni N, Champagne FA, D'Alessio AC, Sharma S, Seckl JR et al. Epigenetic programming by maternal behavior. Nat Neurosci 2004; 7: 847-854.

15. Weaver IC, D'Alessio AC, Brown SE, Hellstrom IC, Dymov S, Sharma S et al. The transcription factor nerve growth factor-inducible protein a mediates epigenetic programming: altering epigenetic marks by immediate-early genes. J Neurosci 2007; 27: 1756-1768.

16. de Kloet ER, Joels M, Holsboer F. Stress and the brain: from adaptation to disease. Nat Rev Neurosci 2005; 6: 463-475.

17. Liu D, Diorio J, Tannenbaum B, Caldji C, Francis D, Freedman A et al. Maternal care hippocampal glucocorticoid receptors, and hypothalamic-pituitary-adrenal responses to stress. Science 1997; 277: 1659-1662

18. Oberlander TF, Weinberg J, Papsdorf M, Grunau R, Misri S, Devlin AM. Prenatal exposure to maternal depression, neonatal methylation of human glucocorticoid receptor gene (NR3C1) and infant cortisol stress responses. Epigenetics 2008; 3: 97-106.

19. McGowan PO, Sasaki A, D'Alessio AC, Dymov S, Labonte B, Szyf M et al. Epigenetic regulation of the glucocorticoid receptor in human brain associates with childhood abuse. Nat Neurosci 2009; 12: 342-348.

20. Radtke KM, Ruf M, Gunter HM, Dohrmann K, Schauer M, Meyer A et al. Transgenerational impact of intimate partner violence on methylation in the promoter of the glucocorticoid receptor. Transl Psychiatry 2011; 1, e21; doi:10.1038/tp.2011.21.
21. Leserman J. Sexual abuse history: prevalence, health effects, mediators, and psychological treatment. Psychosom Med 2005; 67: 906-915.

22. Perroud N, Uher R, Dieben K, Nicastro R, Huguelet P. Predictors of response and drop-out during intensive dialectical behavior therapy. J Pers Disord 2010; 24: 634-650.

23. Preisig M, Fenton BT, Matthey ML, Berney A, Ferrero F. Diagnostic interview for genetic studies (DIGS): inter-rater and test-retest reliability of the French version. Eur Arch Psychiatry Clin Neurosci 1999; 249: 174-179.

24. First M, Gibbon M, Spitzer R, Williams JBW, Smith Benjamin L. Structured Clinical Interview for DSM-IV Personality Disorders (SCID-II). American Psychiatric Association: Washington (DC), 1994.

25. Beck AT, Steer RA, Ball R, Ranieri W. Comparison of beck depression inventories -IA and II in psychiatric outpatients. J Pers Assess 1996; 67: 588-597.

26. Bernstein DP, Fink L. Childhood Trauma Questionnaire. A Retrospective Self-Report. The Psychological Corporation: San Antonio, USA, 1998

27. Sheehan DV, Lecrubier $Y$, Sheehan $K H$, Amorim $P$, Janavs J, Weiller $E$ et al. The miniinternational neuropsychiatric interview (M.I.N.I.): the development and validation of a structured diagnostic psychiatric interview for DSM-IV and ICD-10. J Clin Psychiatry 1998; 59(Suppl 20): 22-33; quiz 34-57.

28. Stouder C, Deutsch S, Paoloni-Giacobino A. Superovulation in mice alters the methylation pattern of imprinted genes in the sperm of the offspring. Reprod Toxicol 2009; 28: 536-541.

29. Teicher MH, Tomoda A, Andersen SL. Neurobiological consequences of early stress and childhood maltreatment: are results from human and animal studies comparable? Ann NY Acad Sci 2006; 1071: 313-323.

30. Teicher MH, Samson JA, Polcari A, McGreenery CE. Sticks, stones, and hurtful words: relative effects of various forms of childhood maltreatment. Am J Psychiatry 2006; 163 . 993-1000.

31. Heim C, Nemeroff CB. The role of childhood trauma in the neurobiology of mood and anxiety disorders: preclinical and clinical studies. Biol Psychiatry 2001; 49: 1023-1039.

32. Fergusson DM, Horwood LJ, Lynskey MT. Childhood sexual abuse and psychiatric disorder in young adulthood: II. Psychiatric outcomes of childhood sexual abuse. J Am Acad Child Adolesc Psychiatry 1996; 35: 1365-1374.

33. Plotsky PM, Thrivikraman KV, Nemeroff CB, Caldji C, Sharma S, Meaney MJ. Long-term consequences of neonatal rearing on central corticotropin-releasing factor systems in adult male rat offspring. Neuropsychopharmacology 2005; 30: 2192-2204.

34. Higley JD, Hasert MF, Suomi SJ, Linnoila M. Nonhuman primate model of alcohol abuse: effects of early experience, personality, and stress on alcohol consumption. Proc Natl Acad Sci USA 1991; 88: 7261-7265.

35. Yates TM. The developmental consequences of child emotional abuse: a neurodevelopmental perspective. J Emotional Abus 2007; 7: 9-34.

36. LaPrairie JL, Heim C, Nemeroff CB. Neuroendocrine effects of early life trauma. In: Lanius RA, Vermetten E, Pain C (eds). The Impact of Early Life Trauma on Health and Disease. The Hidden Epidemic. Cambridge University Press: Cambridge, UK, 2010.

37. De Bellis MD, Keshavan MS, Shifflett $\mathrm{H}$, lyengar S, Beers SR, Hall J et al. Brain structures in pediatric maltreatment-related posttraumatic stress disorder: a sociodemographically matched study. Biol Psychiatry 2002; 52: 1066-1078.

38. Francis D, Diorio J, Liu D, Meaney MJ. Nongenomic transmission across generations of maternal behavior and stress responses in the rat. Science 1999; 286: 1155-1158.

39. Bateman A, Fonagy P. Psychotherapy for Boderline Personality Disorder. Mentalization Based Treatment. Oxford University Press: Oxford, 2004.

40. McGowan PO, Suderman M, Sasaki A, Huang TC, Hallett M, Meaney MJ et al. Broad epigenetic signature of maternal care in the brain of adult rats. PLOS One 2011; 6: e14739.

41. Turner JD, Muller CP. Structure of the glucocorticoid receptor (NR3C1) gene $5^{\prime}$ untranslated region: identification, and tissue distribution of multiple new human exon 1. $\mathrm{J} \mathrm{Mol}$ Endocrinol 2005; 35: 283-292.

42. Philibert R, Caspers K, Beach SRH, Bakermans-Kranenburg MJ, van IJzendoorn MH Reply to: epstein-barr virus transformed DNA as a source of false positive findings in methylation studies of psychiatric conditions. Biol Psychiatry 2011; 70: e27-e28.

Translational Psychiatry is an open-access journal published by Nature Publishing Group. This work is licensed under the Creative Commons Attribution-Noncommercial-No Derivative Works 3.0 Unported License. To view a copy of this license, visit http://creativecommons.org/licenses/by-nc-nd/3.0/

\section{Supplementary Information accompanies the paper on the Translational Psychiatry website (http://www.nature.com/tp)}

